\title{
Monochorionic twin pregnancies increases perinatal risk and is a high risk factor of selective intrauterine growth restriction
}

Jie Chen

Zhujiang Hospital,Southern Medical University

Yue Lian Yang (D 61850306@qq.com )

Zhujiang Hospital https://orcid.org/0000-0002-3618-3326

\section{Research article}

Keywords: Monochorionic diamniotic, Monochorionic twin pregnancies, Selective intra-uterine growth restriction

Posted Date: August 3rd, 2019

DOI: https://doi.org/10.21203/rs.2.12399/v1

License: (c) (i) This work is licensed under a Creative Commons Attribution 4.0 International License.

Read Full License 


\section{Abstract}

Background Twins pregnancy can cause a lot of disease, especially monochorionic twin pregnancies, the prenatal infant will have many diseases and have high mortality rate. According to analysis and compare of the twin pregnancy, especially pregnant woman and puerpera's situation and complication and baby's situation; we hope we can find the reason which causes the fetus growth restrain of monochorionic twin pregnancies. So we can provide some reference for the prenatal health care, complication prevention and prenatal outcome. Methods We divided 489 cases of twin pregnancies into two groups: monochorionic twin and dichorionic twin and compared the clinical features of them. At last, we used the logistic regression analysis method to analyze the risk factors of selective intrauterine growth restriction(sIUGR). Results The incidences of premature rupture of membranes and sIUGR were significant higher in monochorionic twin and twin-twin transfusion syndrome (TTTS) only exists in monochorionic twin. The weight of the newborn babies(both big and small babies)were significant lower in Monochorionic twin. The neonatal transfer rate was significant higher in monochorionic twin. Gestational weeks and weight of newborn babies are the high risk factors of sIUGR. Conclusions The type of chorion has a great influence to the pregnant period and the ending of maternal women. Monochorionic is a high risk factor of the sIUGR, which means that the main cause of sIUGR is from placenta, so it is a kind of "placental origin disease".

\section{Background}

When there are two babies in the uterine cavity of a pregnant woman, we usually call this kind of pregnancy "twin pregnancies". Hellin[1]once calculated that the rate of multiple pregnancies was 1:89"-

${ }^{1}$ ( $\mathrm{n}$ referring to the number of babies). While as to the use of assisted reproductive technology (ART) and the use of ovulation medicine, the rate of multiple pregnancies has been increasing in recent years.

Maternal complications and risk of adverse pregnancy outcomes are higher in twin pregnancies than in singleton pregnancy, so as to the rate of premature birth and perinatal mortality. Twin pregnancies are much easier to get complications, especially monochorionic twin who will get higher rate of mortality and complication. Our research used retrospective way to analyze and compare twin pregnancies, especially the situation of pregnancy women and newborn babies, clinical characteristics and complications between monochorionic twin and dichorionic twin. We hoped to find out the high risk factors of the fetal growth restriction in order to make a reference to the perinatal health care and perinatal outcome of monochorionic twin.

\section{Methods}

\section{Research object}

We collected the cases of twin pregnancies in Zhujiang Hospital of Southern Medical University during Jan.2013 and Dec. 2017. Then we analyzed the basic information, clinical characteristics, complications, 
and perinatal outcomes of twin pregnancies. All the information was collected from the medical records database of Zhujiang Hospital, which was in strict confidence and approved by the Ethics Committee at the Zhujiang Hospital of Southern Medical University.

There were 12948 women giving birth during Jan. 2013 and Dec. 2017 in Zhujiang Hospital, among which 864 cases are twin pregnancies and 489 cases were used in our research.

\section{Case diagnosis standard and condition}

(1)The gestational weeks of all cases were more than 28 weeks and the chorionic situations were defined. The cases which the chorionic situation couldn't be defined, or the gestational weeks were less than 28 weeks, or multiple pregnancy after reduction surgery couldn't be enrolled. (2)We defined the kinds of chorion through the number of fertilized eggs during 6 and 9 weeks and the shape between the placenta and amnion during 10 and 14 weeks by ultrasound. After delivery, we defined it through the placental situation and the result of pathological examination. (3)We checked the gestational weeks of all women in hospital. (4)Fertilization way was divided into natural way and ART which included external fertilization, artificial fertilization and ovulation induction treatment. (5)We checked if puerperae had adverse pregnancy history which included more than two times abortion, embryo damage, fetal anomaly, fetal death and postpartum hemorrhage. (6)Delivery way was divided into natural birth and surgical delivery. (7)Diagnosis of sIUGR was based on an estimated fetal weight (EFW) in one twin of < 10th percentile by ultrasound test. (8)TTTS was diagnosed requiring the presence of the following criteria:

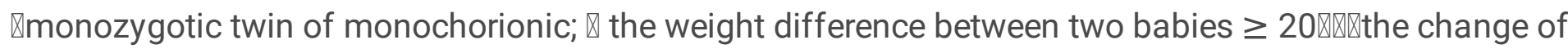
amniotic fluid, the deepest depth of one baby $\otimes 8 \mathrm{~cm}$,the deepest depth of the other baby $\otimes 2 \mathrm{~cm}$.

\section{Research method}

We used retrospective clinical analysis and multiple-factor Logistic regression analysis to analyze 489

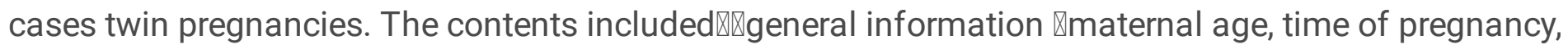
parity, the number of antenatal care, gestational weeks, fertilization way, the time of prenatal hospital

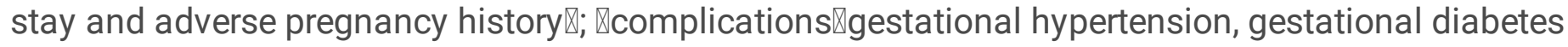
mellitus, intrahepatic cholestasis of pregnancy, late abortion, premature rupture of membranes, premature delivery, anemia, placental abruption, placenta previa , sIUGR, postpartum hemorrhage冈occurrence rate; \the situation of the newborn babies $₫$ weight, length, gender, neonatal transfer rate, asphyxia of the

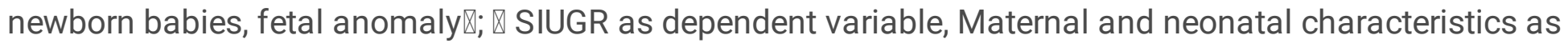
the independent variable, used the multiple-factor logistic regression analysis to analyze the high risk factors of sIUGR (Table 1).

\section{Statistical method}

We used SPSS 16.0 statistical software to do the analysis, used $X^{2}$ test to compare the enumeration date, used average number \pm standard deviation()to show the measurement data and use $t$ test, Mann-Whitney $\mathrm{U}$ test, Chi-square test to compare the clinical data of different kinds of choironic pregnancy. At last, put 
the possible factors which can affect the occurrence and change of sIUGR into the logistic regression analysis. We analyzed the possible factors which will affect the occurrence of sIUGR. A value of $P<0.05$ was selected as the level of significance.

\section{Results}

\section{Compare general information}

As shown in Figure 1, 12948 women giving birth during Jan. 2013 and Dec. 2017 in Zhujiang Hospital and 864 cases were multiple pregnancies. Of these, 375 cases were excluded for data incomplete. Ultimately, 489 cases met all requirements. We divided the 489 cases into two groups according to the types of chorion. The age of pregnant women, the incidence of ART and surgical delivery way were significant lower in Monochorionic twin (Table 2).

\section{Compare the complications}

The incidences of premature rupture of membranes and sIUGR were significant higher in monochorionic twin and TTTS only existed in monochorionic twin (Table 3).

\section{Compare the perinatal infant outcome}

The weight of the newborn babies $\llbracket$ both big and small babies $₫$ was significant lower in Monochorionic twin. The neonatal transfer rate was significant higher in monochorionic twin (Table 4).

\section{Analyze the high risk factors of sIUGR}

We found that gestational weeks and the weight of newborn babies are the high risk factors of sIUGR (Table 5).

\section{Discussion}

The rate of multiple-pregnancy is about $1.1 \mathrm{Q} \otimes 1.2 \mathrm{Z}$, among which the rate of monochorionic twin is about

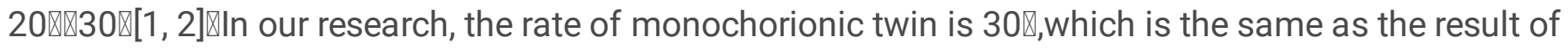
previous researches. Comparing single-pregnancy and dichorionic twin, monochorionic twin had worse perinatal outcomes, the rate of perinatal mortality in monochorionic twin is twice as that in dichorionic twin, and four times as that in single-pregnancy[3]. The rate of fetal abortion during 10-24 weeks of pregnancy in monochorionic twin is six times of that in dichorionic twin and single-pregnancy[4]. The growing environment of monochorionic twin in the utero is very complicated. Monochorionic twins will increase the burden of pregnant women, and they have more risk factors, such as premature delivery, perinatal mortality, fetal growth restriction, congenital malformation, and all of these risk factors will appear earlier and more serious than single-pregnancy. 
SIUGR is one of the most common complications in multiple-pregnancy. It has a high rate of adverse pregnancy outcomes and can affect the cardiovascular function and endocrine system of newborn babies and even can have a great influence after they grow up[5]. Because there were not adequate researches about the pathogenesis of this disease, we didn't get consensus of the treatment and diagnosis of monochorionic twin. The research of sIUGR becomes very popular among complicated multiple pregnancy. SIUGR can happen both in dizygotic twin and monozygotic twin, but the reason of them is different. When sIUGR happens in dichorionic twin, it is associated with placenta, umbilical cord and congenital malformation of one of the two babies; when it happens in monochorionic twin, it is associated with TTTS, placenta, umbilical cord and babies. In dichorionic twin, the sIUGR usually happens in mid or late gestation. But it is very unpredictable when it happens in monochorionic twin, it can happen in early, mid or late gestation. The more earlier happen or the greater difference in weight between two babies, it will have more serious sequela among babies and higher rate of perinatal mortality. Even though it happens in mid or late gestation when the weight difference between two babies increase, the rate of premature delivery, perinatal asphyxia and death will also increase accordingly.

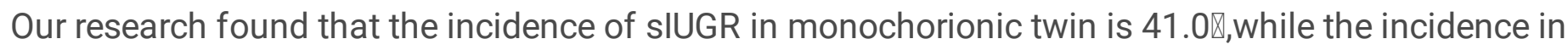
dichorionic twin is 24.1区. The age of pregnant women, the incidence of ART fertilization way and surgical delivery way were significant lower in Monochorionic twin. The incidences of premature rupture of membranes and sIUGR were significant higher in monochorionic twin. The weight of the newborn babies छboth big and small babies $₫$ were significant lower in Monochorionic twin. The neonatal transfer rate was significant higher in monochorionic twin. The result of our research shows: the type of chorion has great influence to the process and the ending of pregnancy; monochorionic twin is the high risk factor of sIUGR, which means the main cause of sIUGR is from placenta, so sIUGR is a kind of "placental origin disease".

The diagnosis standard of sIUGR is still in dispute. At present, the widely recognized standard is brought up by Gratacos[6]区After the ultrasound test, if we find the fetal weight estimation is less than the $10 \%$ of the reference range in relevant gestational weeks, we can diagnose it has slUGR. In monochorionic twin, if either of the fetal weight estimation is less than $10 \%$ of relevant gestational weeks. Prenatal diagnosis and prenatal evaluation of monochorionic twin sIUGR rely on the ultrasound test, including the evaluation of fetal growth, placenta planting position, the test of umbilical artery spectrum and the measurement of amniotic fluid. The test of ultrasound in early gestation includes the type of chorionic twin, the difference of crown rump length of the two babies, placenta planting position, umbilical cord situation. Through these indexes, we can not only find if they have the tendency of getting sIUGR in early ages, but also diagnose if they will get sIUGR in mid or late gestation. The test of ultrasound in mid gestation includes the placenta type, the placenta size, umbilical cord situation and the amount of umbilical vessel. When the files of ultrasound test in early gestation are missing, gender difference of two babies is one of the diagnosis references for the ovulation of twin pregnancy and type of chorion. The diagnosis of TTTS is also very important because both of them are related to the choice of clinical intervention. The difference of amniotic fluid volume between two babies is a very useful index in helping diagnosing whether they have TTTS and sIUGR or not. The ultrasound test in late gestation should pay attention to the infant growth situation, amniotic fluid volume, the umbilical artery blood flow spectrum curve, brain artery blood 
flow spectrum curve. Gratacos $[7,8]$ deemed that there are three kinds of umbilical artery blood flow spectrum curve when sIUGR happens in monochorionic twin pregnancies, it has significant relationship

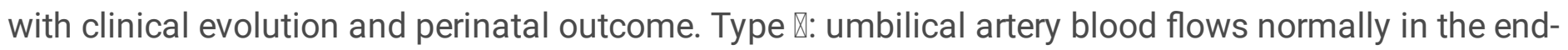

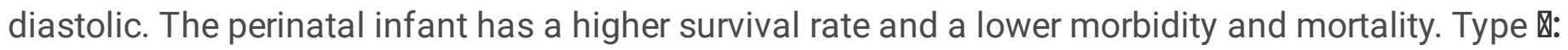
umbilical artery blood flows persistently disappears and inverses in the end-diastolic. This type has the worst prognosis and shortest gestational weeks, the highest rate of intra uterine fetal death and the deterioration of disease, the highest rate of infant mortality rate and the lowest survival rate within 6 months old. Type III: umbilical artery blood flow intermittently disappears and inverses in the enddiastolic. This type has the best clinical consequences.

In conclusion, the perinatal fetal prognosis for monochorionic twin is worse than dichorionic twin, the sIUGR in monochorionic twin has more complicated situation in pregnancy outcome and perinatal infant condition, so we should take close monitoring on them, take the effective interventions promptly and improve the prognosis.

\section{Declarations}

\section{Competing interesting}

The authors declare that they have no competing interests.

\section{References}

1. Deprest JA, Audibert F, Van SD, Hecher K, Mahieucaputo D. Bipolar coagulation of the umbilical cord in complicated monochorionic twin pregnancy. Am J Obstet Gynecol. 2000; 182(2):340-5.

2. Jolly $M$, Taylor $M$, Rose $G$, Govender $L$, Fisk NM. Interstitial laser: a new surgical technique for twin reversed arterial perfusion sequence in early pregnancy. 2001; 108(10):1098-102.

3. Weisz B, Hogen L, Yinon Y, Gindes L, Shrim A. Perinatal outcome of monochorionic twins with selective IUGR compared with uncomplicated monochorionic twins. Am J Obstet Gynecol. 2000; 182:340-5.

4. Quintero RA, Bornick PW, Morales WJ, Allen MH. Selective photocoagulation of communicating vessels in the treatment of monochorionic twins with selective growth retardation. Twin Res Hum Genet. 2011; 14(5):457-62.

5. Hu SJ, Sun H, Deng Y, Lin HF, Ding ZH. Structural analysis of placental vascular system in single placental double pregnancy. Chinese Journal of Practical Gynecology and Obstetrics. 2011; 27(02):134-6.

6. Bennasar M, Eixarch E, Martinez JM, Gratacós E. Selective intrauterine growth restriction in monochorionic diamniotic twin pregnancies. Seminars in Fetal and Neonatal Medicine 2017, 22(6):376-82. 
7. Gratacós E, Antolin E, Lewi L, Martínez JM, Hernandezandrade E. Monochorionic twin with selective intrauterine growth restriction and intermittent absent or reversed end-diastolic flow (Type III): feasibility and perinatal outcome of fetoscopic placental laser coagulation. Ultrasound Obstet Gynecol. 2008; 31(6):669-75.

8. Gratacós E, Lewi L, Oz BM, Acosta-Rojas R, Hernandez-Andrade E. A classification system for selective intrauterine growth restriction in monochorionic pregnancies according to umbilical artery Doppler flow in the smaller twin囚Ultrasound Obstet Gynecol. 2007; 30(1):28-34.

\section{Figure And Table Legend}

Figure 1 Enrollment Flow Diagram

Table 1 Logistic regression model of two classification variable assignment table

Table 2 Comparison of the general data in monochorionic twin and dichorionic twin

Table 3 Comparison of the complications in monochorionic twin and dichorionic twin

Table 4 Comparison of the perinatal infant result in monochorionic twin and dichorionic twin

Table 5 SIUGR influence factors of binary classification logistic regression analysis results $₫ n=460 \bigotimes$

\section{Figures}

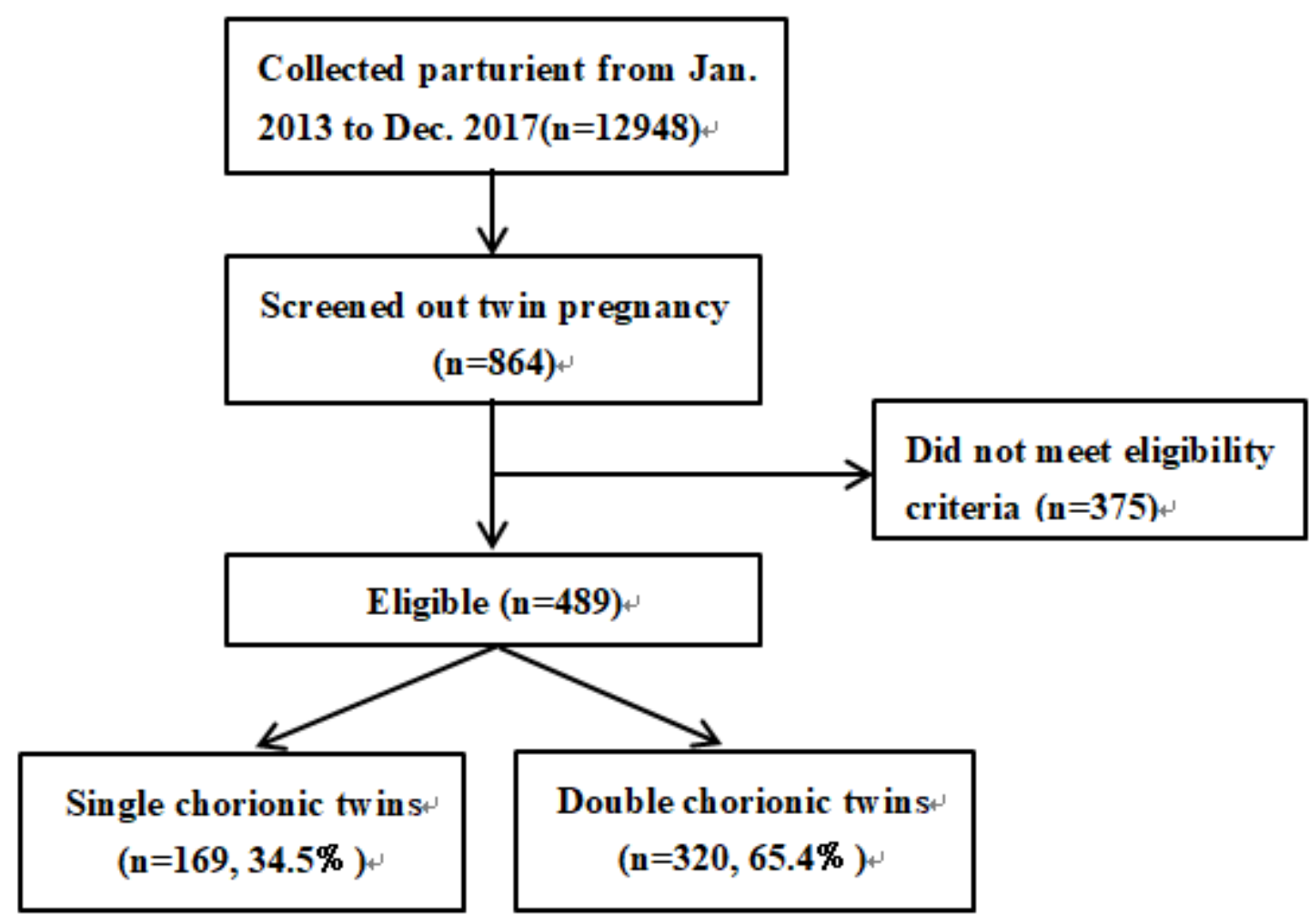


Figure 1

Enrollment Flow Diagram

\section{Supplementary Files}

This is a list of supplementary files associated with this preprint. Click to download.

- supplement1.pdf 